\title{
Fractal Dimension in Animal Model of Alzheimer's Dementia
}

\author{
Ljiljana Martać1 ${ }^{*}$, Slobodan Sekulić \\ ${ }^{1}$ Department of Neurophysiology, Institute for Biological Research “Siniša Stanković”, University of Belgrade, Belgrade, Serbia \\ ${ }^{2}$ Department of Neurology, University Hospital, Clinical Center of Vojvodina, Novi Sad, Serbia \\ Email: *ljmartac@ibiss.bg.ac.rs
}

How to cite this paper: Martać, L. and Sekulić, S. (2018) Fractal Dimension in Animal Model of Alzheimer's Dementia. Journal of Behavioral and Brain Science, 8, 512-518. https://doi.org/10.4236/jbbs.2018.89031

Received: August 16, 2018

Accepted: September 24, 2018

Published: September 27, 2018

Copyright (c) 2018 by authors and Scientific Research Publishing Inc. This work is licensed under the Creative Commons Attribution International License (CC BY 4.0).

http://creativecommons.org/licenses/by/4.0/

\begin{abstract}
The aim of this study was to qualitatively described effect of the oxidative stress, neurotransmission change and the neurodegeneration in animal model of chronic intoxication by aluminum. Electrocortical brain activity of animal model of stress and neurodegeneration is comparable with Alzheimer's Dementia $(\mathrm{AD})$. We used adult animals, during 6 weeks intraperitoneally treated with aluminum. Both animals and patients with Alzheimer's Dementia have increased relative spectral power in delta range. By fractal analysis we described changes in electrocortical activity of aluminum intoxication animals compare to physiological control. We used change in delta range to calculate fractal dimension for the pathophysiological state of disease. We evaluate effect of stress and neurodegeneration, oxidative stress and accumulation of beta amyloid and neurofibrillary tangles as change in fractal dimension (FD). We conclude that change in fractal dimension could be used for prognosis of $\mathrm{AD}$. Results show that decrease in fractal dimension could be used for evaluation of changes in neural activity in occurrence of AD.
\end{abstract}

\section{Keywords}

Neurotoxicity, Fractal Dimension, Alzheimer's Dementia

\section{Introduction}

Alzheimer's disease is characterized by cognitive impairment, progressive neurodegeneration and formation of amyloid- $\beta$ containing plaques and neurofibrillary tangles. The neurodegenerative process in $\mathrm{AD}$ is characterized by synaptic damage accompanied by neuronal loss. Pathology of synapses and defective neurogenesis in $\mathrm{AD}$ are related to progressive accumulation of $\mathrm{A} \beta$ resulting in the formation of toxic oligomers. 
Fractal analysis methods can be used to quantify complexity of brain activity and changes in structure of brain regions. Previous investigations show that patients with $\mathrm{AD}$ have decreased fractal dimension of brain activity [1] [2]. This study compares contribution of different events in change of fractal dimension. Stress events and neurodegeneration are risk factors in occurrence of Alzheimer's Dementia [3]. We used animal model of aluminum intoxication for evaluation of this events [4]. Pathophysiological state of disease achieved by treatment with aluminum includes oxidative stress and neurotransmission disruption. Primary events are change in ion homeostasis and neuron transmission, connected with aluminum accumulation in brain through the blood brain barrier. Secondary events progress in disease are cytotoxicity and neurodegeneration caused by metabolic change in neuron. All this events could be qualitatively described by changes in electrocortical activity and quantitatively described by fractal dimension.

\section{Methods}

All animal procedures were in compliance with Directive 2010/63/EU on the protection of animals used for experimental and other scientific purposes, and were approved by the Ethical Committee for the Use of Laboratory Animals of the Institute for Biological Research "Siniša Stanković", University of Belgrade.

Wistar male rats age 2 months were exposed to aluminum for 6 week. Ten animals were intraperitonealy injected with doses of 2 and $4 \mathrm{mg} / \mathrm{kg}$ of aluminum daily and exposed to stress (all animals are in one cage) and the other group of animals were treated with $6 \mathrm{mg} / \mathrm{kg}$ of aluminum and had neurofibrilary tangles and beta amyloid. After treatment we recorded electrocortical activity of parietal cortex (P: $2.5 \mathrm{~mm}, \mathrm{~L}: 2 \mathrm{~mm}$ in recpect to bregma) and cerebellum cortex (P: 10.5 $\mathrm{mm}, \mathrm{L}: 1.5 \mathrm{~mm}$ in respect to bregma) with tungsten electrodes. Rats were during the experiment light anesthetised by Nembutal and immobile. Recorded were done for $121 \mathrm{~s}$ in intervals of 5 min during 2 hours with sampling rate $256 \mathrm{~Hz}$.

In brief, we calculated FD using Higuchi's algorithm [5], fractal dimension values of cerebral electrocortical activity in anesthetized rats. It was supposed that a series of cortical activity was a time sequence $x(1), x(2), \ldots, x(N)$ and we constructed $k$ new self-similar time series $x(k, m)$ for $m=1,2, \ldots, k, k=1, \ldots$, $k_{\max }$. The length $L(m, k)$ was computed for each of the $k$ time series or curves $x(k, m) . L(m, k)$ was averaged for all $m$ forming the mean value of the curve length $L(k)$ for each $k$. An array of mean values $L(k)$ was obtained and the FD was estimated: $\mathrm{FD}=\log (L(k)) / \log (1 / k)$. Parameter $N=200$ was within the range used by other authors and was equivalent to an epoch (window) duration of $0.8 \mathrm{~s}$ at the sampling rate of $256 \mathrm{~s}^{-1}$. We have chosen $k_{\max }=8$ on the basis of our recent study [6] on the optimum choice of $k_{\max }$ value. Individual FD values were averaged across all epochs for particular experimental conditions to obtain the mean FD. Fractal analysis was performed for electrocortical signals of animals with different types of pathophysiological state. Means and variances of FD of 
signals obtained under particular experimental conditions were calculated using MATLAB routines and tested by student's t-test. We calculated FD for animal model and used increase in delta relative spectral power to approximate results on pathophysiological state in $\mathrm{AD}$. All events in developing the disease shows decrease in fractal dimension.

\section{Results}

Table 1 described effect of the oxidative stress, neurotransmission change and the neurodegeneration in animal model of chronic intoxication by aluminum. We compare group of aluminum treated animals with control, untreated group of animals. Fractal dimension after aluminum treatment shows decrease. Also we find differences between FD for cerebrum and cerebellum.

Previous results shows that decrease in fractal dimension can be measure of stress and neurodegeneration due to aluminum intoxication. However these changes are different for brain structures due to mechanisms of plasticity and accumulation. Linearisation shows changes is fractal dimension which correspond effect of stress and neurodegeneration in animal model of aluminum intoxication. Both in animals and humans increase in delta spectral range could be reliable for pathophysiological state of disease.

Figure 1(a) \& Figure 1(b) presents FD in control group and group of aluminum treated animals in cerebrum and cerebellum. Effect of stress and neurodegeneration is comparable with $\mathrm{AD}$ and change in $\mathrm{FD}$ is present for animal and $\mathrm{AD}$. Linearisation shows change in FD due to stress and neurodegeneration (Figure 2).

Oxidative stress in intoxication shows decrease in FD. We used change in relative spectral power in delta range to approximate this effect on $\mathrm{AD}$. We notice change in FD between cerebrum and cerebellum but the molecular mechanism of oxidative stress is the same for cerebrum and cerebellum and present as linearisation.

Figure 3 shows decrease of fractal dimension on cerebrum and cerebellum in state of cause comparable with occurrence of Beta amyloid and neurofibrilary

Table 1. Fractal dimension in group of 10 control and 10 treated animals with aluminum.

\begin{tabular}{ccccc}
\hline & cerebrum 1. & cerebrum r. & cerebellum 1. & cerebellum r. \\
\hline Fd control & & & & \\
averige & 1.388 & 1.377 & 1.505 & 1.448 \\
SD & 0.523 & 0.068 & 0.112 & 0.131 \\
& & & & \\
\multicolumn{2}{l}{ Fd aluminium } & & & \\
averige & 1.303 & 1.325 & 1.457 & 1.448 \\
SD & 0.049 & 0.077 & 0.098 & 0.087 \\
\hline
\end{tabular}




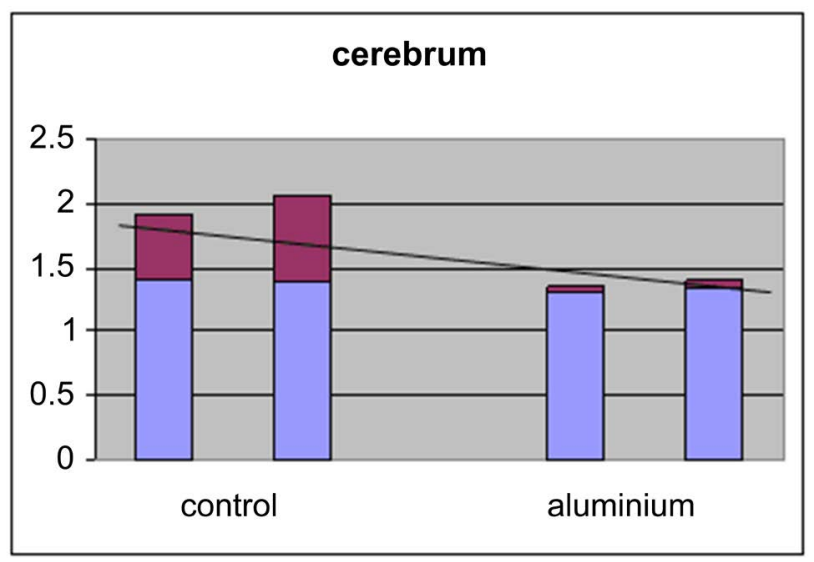

(a)

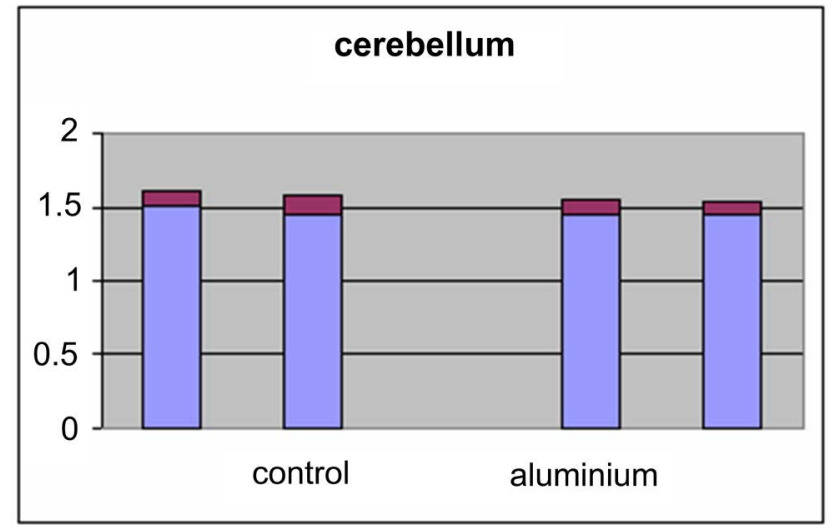

(b)

Figure 1. (a) \& (b): Fractal dimension in cerebrum and cerebellum in control condition and intoxication (animal/AD).

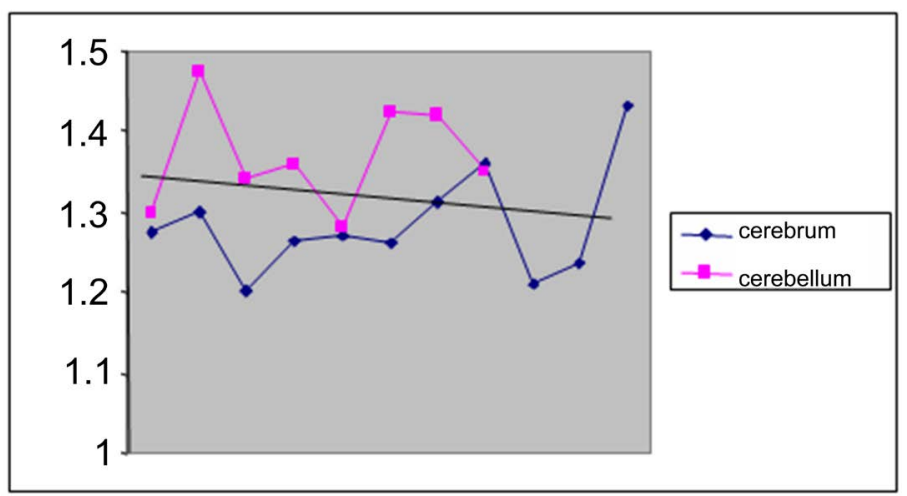

Figure 2. Fractal dimension in animal model with increased rel.sp.power in delta rangeeffect of oxidative stress.

tangles in Alzheimer's Dementia. Due to selectively accumulation in brain we have fluctuation in cerebrum and response in cerebellum due to connectivity with cerebral regions.

We can conclude that stress and neurodegeneration as primary events of neurotoxicity in aging and occurrence of beta amyloid and neurofibrilary tangles 


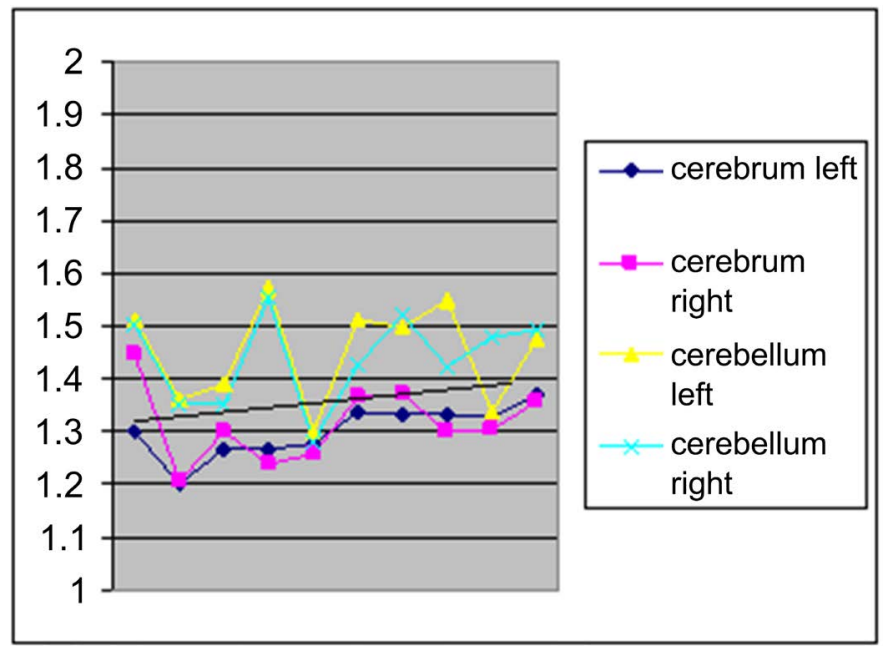

Figure 3. Fractal dimension in rats with Beta amyloid and neurofibrilary tangles.

as secondary effect, both have decreased fractal dimension. Both events are responsible for development of Alzheimer's disease.

\section{Discussion}

This animal model is convenient because stress and neurodegeneration have synergistic effect in diagnosis and prognosis of Alzheimer's Dementia. In adult types of intoxication is accompanied by reduced apoptosis and neurodegeneration [7]. Due to the accumulation of aluminum in the brain leads to oxidative stress and inflammation of the brain tissue, which results in the development of neurodegeneration [8]. Such an animal model is comparable with Alzheimer's disease [9].

At the level of cerebrum as Table 1 shows decrease in fractal dimension as a measure of pathophysiological state. Due to mechanisms of recovery which are still unknown we can observe increase in fractal dimension which is in animal model different compare to humans so this results should be observed as the mean value which describes typical pathphysiological state and might be used in diagnosis. Lower value compare to mean value could be estimate as progression of disease. Higher values compare to control are due to mechanisms of adaptation in animal and can not be used for humans. However the degree of max adaptation compare to mean value could be indicator of tolerance because in experiments all animals survive treatment.

Comparation shows that fractal dimension in the group of animals with neurofibrilary tangles and beta amyloid (Figure 2) only in cerebrum might have higher fractal dimension. However changes of fractal dimension in cerebrum are due to mechanisms which are still not resolved [10]. It might be interesting that stress and neurodegeneration have opposite effects in Alzheimer's disease compare to effects of molecular occurrence of beta amyloid and neurofibrilary tangles. Fractal dimension can be used in diagnosis and prognosis of Alzheimer's Dementia. 
Our results shows that stress and neurodegeneration, oxidative stress due to toxicity and accumulation of beta amyloid and neurofibrilary tangles have decreased FD but the effect of these events have different molecular mechanisms. This study presents contribution of all this events as decrease of FD.

\section{Conclusion}

Decrease in fractal dimension in this animal model of $\mathrm{AD}$ could be used for diagnosis and prognosis of Alzheimer's Dementia. Study shows that cerebrum and cerebellum have differences in FD so we might conclude that response to toxication and plasticity mechanisms are different for this structure. State of dementia and mental activity are presented as a change in delta activity.

\section{Acknowledgements}

This work was supported by the Ministry of Education, Science and Technological Development of the Republic of Serbia (project 175006).

\section{Conflicts of Interest}

The authors declare no conflicts of interest regarding the publication of this paper.

\section{References}

[1] Jurki, T. and Kuikka, M.D. (2002) Fractal Analysis of Cerebral Blood Flow Distribution in Alzheimer's Disease. Journal of Nuclear Medicine, 43, 1727-1728.

[2] King, R.D., Brown, B., Hwang, M., Jeon, T. and George, A.T. (2010) Fractal Dimension Analysis of the Cortical Ribbon in Mild Alzheimer's Disease. Neuroimage, 53, 471-479. https://doi.org/10.1016/j.neuroimage.2010.06.050

[3] Martać, L.J., Podgorac, J., Sekulić, S. and Čapo, I. (2014) Animal Model of Neurodegeneration and Stress Cause by Aluminium Toxicity. American Journal of Bioscience, 2, 28-31.

[4] Martać, L.J., Kesić, S., Ćulić, M., Grbić, G., Spasić, S., Sekulić, S. and Lalošević, D. (2006) Effect of Aluminium Neurotoxicity on Rat Brain Activity. Acta Physiologica and Pharmacologica Serbica, 42, 219-225.

[5] Higuchi, T. (1988) Approach to an Irregular Time Series on the Basis of the Fractal Theory. Physics D, 31, 277-283. https://doi.org/10.1016/0167-2789(88)90081-4

[6] Spasic, S., Kalauzi, A., Culic, M., Grbić, G. and Martać, L.J. (2005) Estimation of Parameter $\mathrm{k}_{\max }$ in Fractal Analysis of Rat Brain Activity. Annals of the New York Academy of Sciences, 1048, 427-429. https://doi.org/10.1196/annals.1342.054

[7] Bharathi, P., et al. (2008) Molecular Toxicity of Aluminum in Relation to Neurodegeneration. Indian Journal of Medical Research, 128, 545-556.

[8] Campbell and Bondy, S.C. (2010) Aluminum Induced Oxidative Events and Its Relation to Inflammation: A Role for the Metal in Alzheimer's Disease. Cellular and Molecular Biology, 46, 721-730.

[9] Kawahara, M. (2005) Effects of Aluminum on the Nervous System and Its Possible Link with Neurodegenerative Diseases. Journal of Alzheimer's Disease, 8, 171-182. https://doi.org/10.3233/JAD-2005-8210 
[10] Gupta, V.B., Anltha, S., Hegde., M.L., Zecca, L., Garruto, R.M., Ravid, R., Shankar, K., Stein, R., Shanmugavelu, P. and Rao, K.S.J. (2005) Aluminum in Alzheimer's Disease: Are We Still at a Crossroad. Cellular and Molecular Life Sciences, 62, 143-158. https://doi.org/10.1007/s00018-004-4317-3 